ARTICLE

\title{
High-resolution patterning of colloidal quantum dots via non-destructive, light-driven ligand crosslinking
}

Jeehye Yang 1,9, Donghyo Hahm²,9, Kyunghwan Kim (10) 3,9, Seunghyun Rhee ${ }^{3}$, Myeongjae Lee (i) 4 ,

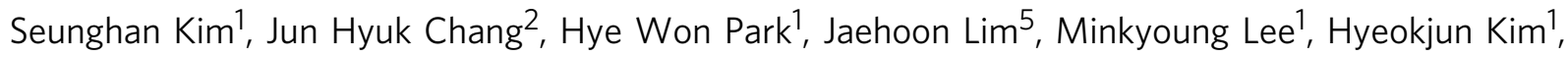
Joohee Bang ${ }^{6}$, Hyungju Ahn ${ }^{6}$, Jeong Ho Cho ${ }^{7}$, Jeonghun Kwak (i) ${ }^{3}$, BongSoo Kim ${ }^{8}$, Changhee Lee (i) ${ }^{3}$, Wan Ki Bae (i) ${ }^{2 \otimes} \&$ Moon Sung Kang (i) ${ }^{1 \times}$

Establishing multi-colour patterning technology for colloidal quantum dots is critical for realising high-resolution displays based on the material. Here, we report a solution-based processing method to form patterns of quantum dots using a light-driven ligand crosslinker, ethane-1,2-diyl bis(4-azido-2,3,5,6-tetrafluorobenzoate). The crosslinker with two azide end groups can interlock the ligands of neighbouring quantum dots upon exposure to UV, yielding chemically robust quantum dot films. Exploiting the light-driven crosslinking process, different colour CdSe-based core-shell quantum dots can be photo-patterned; quantum dot patterns of red, green and blue primary colours with a sub-pixel size of $4 \mu \mathrm{m} \times 16 \mu \mathrm{m}$, corresponding to a resolution of $>1400$ pixels per inch, are demonstrated. The process is non-destructive, such that photoluminescence and electroluminescence characteristics of quantum dot films are preserved after crosslinking. We demonstrate that red crosslinked quantum dot light-emitting diodes exhibiting an external quantum efficiency as high as $14.6 \%$ can be obtained.

\footnotetext{
${ }^{1}$ Department of Chemical and Biomolecular Engineering, Sogang University, Seoul 04107, Republic of Korea. ${ }^{2}$ SKKU Advanced Institute of Nanotechnology (SAINT), School of Nano Science \& Technology, Sungkyunkwan University (SKKU), Suwon 16419, Republic of Korea. ${ }^{3}$ Department of Electrical and Computer Engineering, Inter-University Semiconductor Research Center, Seoul National University, Seoul 08826, Republic of Korea. ${ }^{4}$ Department of Chemistry, Korea University, Seoul 02841, Republic of Korea. ${ }^{5}$ Department of Energy Science, Center for Artificial Atoms, Sungkyunkwan University (SKKU), Suwon 16419, Republic of Korea. ${ }^{6}$ Pohang Accelerator Laboratory, POSTECH, Pohang 37673, Republic of Korea. ${ }^{7}$ Department of Chemical and Biomolecular Engineering, Yonsei University, Seoul 03722, Republic of Korea. ${ }^{8}$ Department of Chemistry, Ulsan National Institute of Science and Technology (UNIST), Ulsan 44919, Republic of Korea. ${ }^{9}$ These authors contributed equally: Jeehye Yang, Donghyo Hahm, Kyunghwan Kim. ${ }^{凶}$ email: wkbae@skku.edu; kangms@sogang.ac.kr
} 
C olloidal quantum dots (QDs), nanocrystalline semiconductors with dimensions residing in the quantum confinement regime ${ }^{1-4}$, exhibit ultrahigh colour purity and near-unity luminescence quantum yield ${ }^{5-7}$. These excellent characteristics of QDs have led the materials to be successfully incorporated into commercial display devices, positioned to challenge organic light-emitting diode (OLED) technology. These commercial devices, as of now, harness the photoluminescence (PL) characteristics of QDs; PL from QDs in these devices contributes to forming a high-quality white light source for the display, whereby the white light is split into primary colours through liquid crystal layers and colour filters before being conceived by the human eye $\mathrm{e}^{8-10}$. The technology is now driven to launch displays based on the electroluminescence (EL) of QDs ${ }^{11-22}$, akin to what has been achieved from OLEDs. Among the issues to be resolved, the development of processing methods to precisely locate red $(R)$, green $(G)$ and blue $(B)$ QDs at a given position in the pixel over a large area is one of the critical challenges in realising EL displays based on QDs. The challenge arises from the fact that QDs are processed from solutions, unlike organic luminophores used for OLEDs that are typically processed by thermal evaporation ${ }^{23-25}$. While the solution processability of QDs allows low-cost production of films over a large area, it prevents conducting a secondary solution process on top of the underlying QD film. This indicates that conventional photoresistbased patterning methods are hardly applicable, unless the surface of QDs is delicately managed ${ }^{26}$; the QD films would be soluble to the solvent used to apply the photoresist. Furthermore, forming patterns of different colour QDs (e.g., $R, G, B$ patterns of QDs) side-by-side by conducting consecutive cycles of solution processing is challenging, because processing one of the QD layers is likely to damage the underlying QD patterns. Han and colleagues $^{27}$ employed an additional layer of positively charged polyelectrolyte underneath the film of QDs modified with negatively charged ligands. High-resolution patterns of QDs could be successfully prepared, but the luminescence characteristics of QDs could not be preserved completely. Alternative patterning methods for QD films have been developed extensively, including ink-jet printing $28-30$ and micro-contact printing ${ }^{31-33}$. These methods, however still require further development for industrial-scale usage in terms of the achievable uniformity, resolution and throughput rate.

The utilisation of light-driven chemical/physical transformation of QD films for patterning is a promising strategy that can meet these practical requirements. Manna and colleagues ${ }^{34}$ demonstrated that aliphatic ligands of QDs can be activated under X-ray exposure to form chemically crosslinked QD films. A similar approach was done by Liao and colleagues ${ }^{35}$ using an $\mathrm{Ar}$ plasma as the irradiation source. Despite the success of patterning, the use of a high-energy X-ray or plasma source is likely to cause loss of PL, which prevents the use of this process for luminescent applications. Talapin and colleagues ${ }^{36,37}$ designed inorganic ligand molecules anchored on the surface of QDs, which can be transformed upon exposure to various wavelengths of ultraviolet (UV)-Visible (Vis) light (254-450 nm) and even to $e$-beam. As the solubility of the QD film alters as the surface properties of ligand molecules change under irradiation, QD film could be patterned by selectively removing either the irradiated region or the un-exposed region with an appropriate developer solvent. Consequently, they demonstrated micrometre-sized QD patterns and multi-layered patterns of $R G B$ QDs by repeating the patterning process. However, the luminescence properties of the resulting QD patterns have not been investigated comprehensively, which are critical to their optical or optoelectronic applications.
Here, we report a simple yet effective method to form highresolution patterns of QDs that preserves the inherent luminescent properties of the material using a light-driven ligand crosslinker (LiXer). UV exposure on a blended film prepared from QD-LiXer mixed solutions galvanises the chemical reaction between azides and the alkyl chain of QD surface ligands to construct a chemically robust QD network. Because of the excellent crosslinking efficiency of fluorinated phenyl azides we used $^{38-40}$, QD patterns are readily achieved with a small amount of LiXer (less than $5 \mathrm{wt} \%)$ using a handheld UV-lamp (254 nm, $0.4 \mathrm{~mW} \mathrm{~cm}^{-2}$ ) over a short period of time $(5 \mathrm{~s})$. As the resulting crosslinked QD films are structurally robust against subsequent solution processes, multiple patterns of QDs can be formed through consecutive cycles of solution-based film deposition and photo-patterning processes. Based on this strategy, we successfully fabricate QD line patterns with a minimum feature size of $3 \mu \mathrm{m}$ and $R G B$ QD patterns with a sub-pixel size of $4 \mu \mathrm{m} \times 16 \mu \mathrm{m}$ that corresponds to a resolution of $>1400$ pixels per inch (p.p.i.). Owing to the little contents of LiXer and benign processing conditions, degradation in the PL characteristics of QDs during the patterning process and the associated EL characteristics of the QD-LEDs could be avoided. Consequently, QD-LEDs yielding an external quantum efficiency (E.Q.E.) of $14.6 \%$ could be obtained from the crosslinked QD layer, which is a comparable value achievable from pristine QD layer. The simple strategy presented here will make a significant impact on the production of highresolution, large area, full-colour QD-LEDs, which are intensively explored across the scientific community to industry.

\section{Results}

Description of the photo-patterning method based on LiXer. Figure 1 describes the core of the high-resolution photo-patterning method for QDs. The method utilises ethane-1,2-diyl bis (4-azido-2,3,5,6-tetrafluorobenzoate) as the LiXer that contains two fluorinated perfluorophenyl azide groups at both ends of the molecule ${ }^{41-44}$. The chemical structure of ethane-1,2-diyl bis(4azido-2,3,5,6-tetrafluorobenzoate) is shown in Fig. 1a. Fluorinated aryl azide is a well-known photo-active moiety forming reactive nitrene intermediate upon exposure to UV $(254 \mathrm{~nm})$, which can easily undergo $\mathrm{C}-\mathrm{H}$ insertion reaction in the presence of alkyl chains nearby ${ }^{45-47}$. In our scheme, the crosslinker with two fluorinated phenyl azide terminals is intended to undergo $\mathrm{C}-\mathrm{H}$ insertion reaction into the long aliphatic chains of the ligands (i.e., oleic acids or alkyl thiols) that passivate the surface of QDs. Therefore, it allows crosslinking the ligands of neighbouring QDs under exposure to UV. Unlike previous methods ${ }^{36,37}$, the new method can directly utilise high-quality QDs typically terminated with long alkyl chains without undergoing additional ligand modification, which often degrades the luminescence quantum yield of the materials. The patterns of QDs can be formed by (i) simple spin-coating of a solution mixture of QDs and LiXer onto a substrate, (ii) irradiation of UV and (iii) simple developing step using conventional organic solvents (Fig. 1b), all of which are well-established procedures used for photolithography in the semiconductor industry. Moreover, owing to the crosslinked nature of the resulting QD films, they should be chemically robust against subsequent solution processing, even using the very same solvent that was used to cast the given QD films. This allows for a large degree of freedom in realising QD patterns of multiple colours. Since the processing of different coloured QDs can be conducted repeatedly as many times as needed, lateral patterns of $R G B$ QDs (Fig. 1c) as well as the vertical or tandem stacking of different QD layers (Fig. 1d) should also be possible by repeating the procedures. 
a

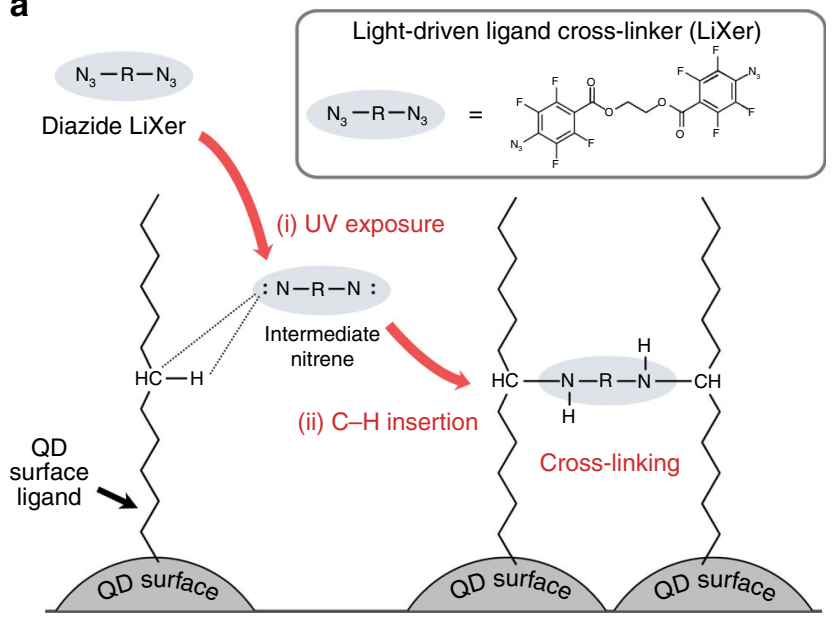

b

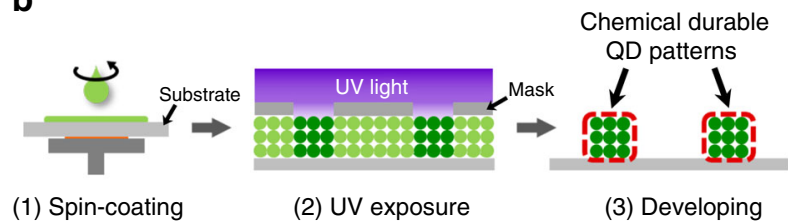

C

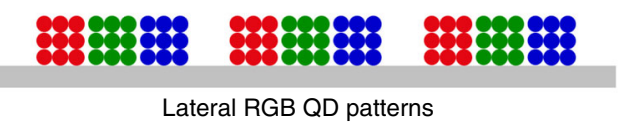

d

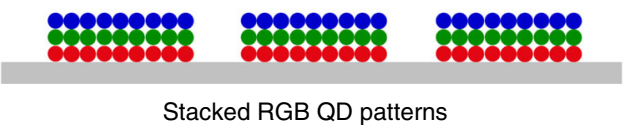

Fig. 1 Schematic illustration of QD patterns using LiXer. a Schematic description of the ligand crosslinking process between neighbouring QDs based on the $\mathrm{C}-\mathrm{H}$ insertion reaction of the nitrene moiety of LiXer. The inset shows the chemical structure of ethane-1,2-diyl bis(4-azido-2,3,5,6-tetrafluorobenzoate), used as the LiXer in this study. b Schematic description of the photo-patterning processes of QDs using LiXer. c Laterally positioned patterns and d vertically stacked patterns of $R G B$ QDs, achievable from consecutive applications of the photo-patterning process.

Figure 2a shows a series of Fourier-transform infrared spectroscopy (FT-IR) spectra for QD films containing the LiXer that was prepared by spin-casting a mixture of $13-\mathrm{nm}$ sized $\mathrm{CdSe} / \mathrm{CdZnS}$ QDs and LiXer (5 wt\%) in toluene. Transmission electron microscope (TEM) images, X-ray diffractograms and absorbance/PL spectra of the QDs used in this work are shown in Supplementary Figs. 1, 2 and 3, respectively. The black spectrum is from the film obtained before it was exposed to UV. The peaks at $2130 \mathrm{~cm}^{-1}$ and $1250 \mathrm{~cm}^{-1}$ reflect the presence of the $\mathrm{N}_{3}$-moieties in the mixture film. The red spectrum is obtained from the same film after UV exposure to a handheld UV source $(254 \mathrm{~nm}$, $0.4 \mathrm{~mW} \mathrm{~cm}^{-2}$ ). Clearly, the peaks associated with the $\mathrm{N}_{3}$-moieties were reduced after the exposure to UV, suggesting that chemical reaction described in Fig. 1a has proceeded. Although the formation of the reactive nitrene intermediate and the final secondary amine moiety resulting from the $\mathrm{C}-\mathrm{H}$ insertion reaction could not be confirmed directly from the FT-IR spectrum $^{43}$, we conjecture that the $\mathrm{N}_{3}$-moieties of LiXer underwent the $\mathrm{C}-\mathrm{H}$ insertion reaction with the long alkyl ligands of neighbouring QDs, which formed the chemically crosslinked QD assembly. Quantitative description on the photon-to-crosslink conversion process of LiXer is provided in Supplementary Information (Supplementary Fig. 4 and Supplementary Discussion). The crosslinking did not reduce the interparticle distance between the QDs. As shown in Fig. 2b, the GI-SAXS pattern of a 13-nm sized CdSe/CdZnS QD added with LiXer (5 wt\%) but not exposed to a UV source showed a peak at $q_{\mathrm{xy}}=0.04386 \AA^{-1}$, corresponding to the mean centre-to-centre distance $(d)$ for neighbouring QDs of $14.3 \mathrm{~nm}$. The same film that underwent the crosslinking reaction (red) also showed a peak with a similar $q_{\mathrm{xy}}$ value $\left(0.04394 \AA^{-1}\right)$, indicating that no discernible change in $d$ has occurred upon the photo-crosslinking process.

Crosslinking the ligands awards the ability to withstand a structural failure against consecutive solution processing to QD films. Figure $2 c$ shows the photographs of the crosslinked versus pristine QD films when dipped into the toluene. Note that toluene was the mother solvent that was used to cast the QD film. The submerged part of the pristine QD film dissolved immediately upon dipping to toluene. By contrast, noticeable change in the crosslinked film was not found even after dipping for a long period (Fig. 2d, e). This supports the idea to devise multicoloured QD patterns either placed side-by-side or stacked vertically simply by repeating the photo-crosslinking reaction of QD films with LiXer and rinsing steps. In fact, only 2 wt\% of LiXer in 13-nm sized CdSe/CdZnS QD solution was sufficient to ensure the structural robustness of the QD film.

Lateral and tandem pattering of QDs. By exploiting the structural robustness of the crosslinked QD films, we could fabricate QD patterns by selective UV exposure through a patterned photomask. Figure 3a, b show sets of optical and fluorescence (inset) images of dot and line patterns based on red QDs, respectively. The patterns were achieved by (i) spin-coating the mixture solution of QD and LiXer in toluene onto a $\mathrm{Si} / \mathrm{SiO}_{2}$ wafer, (ii) exposing the film to UV irradiation $\left(254 \mathrm{~nm}, 0.4 \mathrm{~mW} \mathrm{~cm}^{-2}\right)$ through a photomask and (iii) removing the uncrosslinked regime of the film by rinsing with toluene. Dot patterns with a diameter of $2 \mu \mathrm{m}$ and a spacing of $3 \mu \mathrm{m}$ as well as line patterns with a width of $3 \mu \mathrm{m}$ and a spacing of $4 \mu \mathrm{m}$ could be successfully attained (atomic force microscopy (AFM) image and height profile in Fig. 3a, b). The line edge roughness (LER) of these line patterns was $0.14 \mu \mathrm{m}$ (Supplementary Fig. 5a). Dot and line patterns could also be formed based on green-emitting CdSe/CdZnSeS QDs (Supplementary Fig. 6). LER of these green-emitting QD line patterns was $0.15 \mu \mathrm{m}$ (Supplementary Fig. 5b).

Repeated cycles of QD film deposition, photo-patterning and rinsing processes allowed to achieve well-defined high-resolution patterns of different colour QDs. In fact, one should remember that the development of scalable processing methods to precisely locate $R G B$ QDs in designated positions within a given pixel is one of the critical challenges in realising EL displays based on QDs. Figure 3c, d displays fluorescence images of the resulting $R G B$ QD patterns after repeating photo-patterning steps. The size of a single pattern in the image is $4 \mu \mathrm{m}$ by $16 \mu \mathrm{m}$. The three $R G B$ patterns with a spacing of $2 \mu \mathrm{m}$ constitute a single $18 \mu \mathrm{m}$ by $18 \mu \mathrm{m} R G B$ square pixel, which corresponds to $>1400$ p.p.i. in terms of the display pixel resolution. This value is higher than the value that is currently used in commercial OLED displays. In addition to the patterns placed side-by-side, exploiting the structural robustness of the crosslinked QD film allowed the vertical stacking of different colour patterns of QDs. Figure $3 \mathrm{e}$ shows a fluorescence image of "the three primary colours of light" expressed through $R G B$ QD patterns, formed by repeating the 

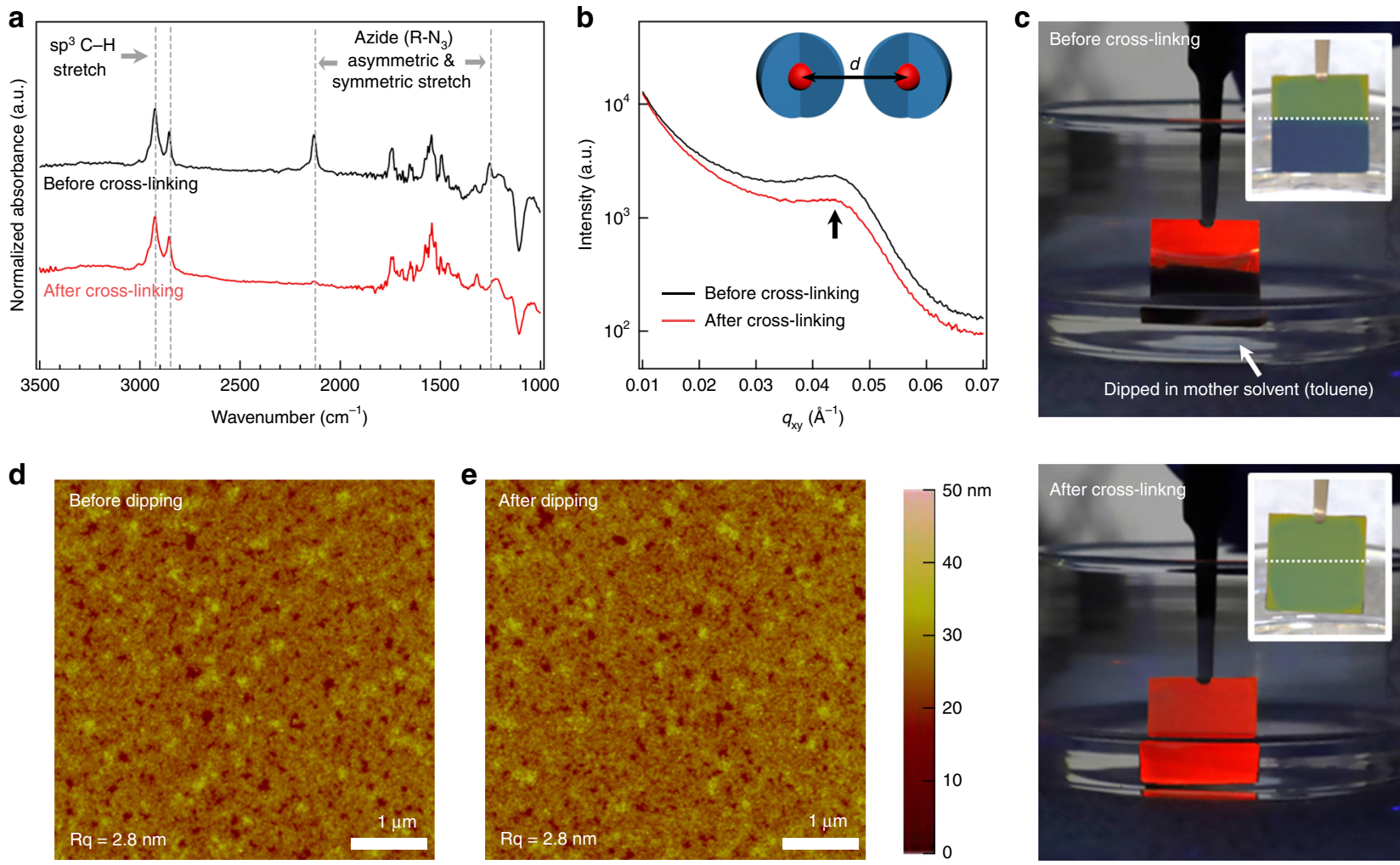

Fig. 2 Characterisation of crosslinked QD films prepared with LiXer. a FT-IR spectra and $\mathbf{b}$ GI-SAXS patterns of 13-nm sized CdSe/CdZnS QD films blended with LiXer ( $5 \mathrm{wt} \%$ ) before (black) versus after (red) UV exposure ( $254 \mathrm{~nm}, 0.4 \mathrm{~mW} \mathrm{~cm}{ }^{-2}$ ). The FT-IR spectra were normalised to the maximum absorbance of the $\mathrm{sp}^{3} \mathrm{C}-\mathrm{H}$ stretch peak at $2924 \mathrm{~cm}^{-1}$ and displayed with an offset for easier comparison. c Photographs of a crosslinked QD film with presence of $5 \mathrm{wt} \%$ LiXer (bottom) and a pristine QD film (top). Both films were partially dipped into toluene, which is the mother solvent that was used to cast these QD films. AFM images of a crosslinked 13-nm sized CdSe/CdZnS QD film $\mathbf{d}$ before and $\mathbf{e}$ after being dipped into toluene.

photo-crosslinking process three times. The resulting pattern nicely demonstrates that the photo-crosslinked QD films can be stacked vertically, as well as horizontally. Furthermore, $\mu$ m-thick layers of QDs can be produced by repeating multiple rounds of film-casting and photo-crosslinking steps, which can be exploited for colour conversion layer applications of QDs (Supplementary Fig. 7).

Photophysical properties of crosslinked QDs with LiXer. The adoption of reactive radicals in patterning QD films is often regarded as a double-edged sword. The low activation energy to generate radicals with high reactivity enables effective chemical crosslinking of QD solids at a mild reaction condition, but simultaneously the reactive radicals could attack the QD surface and create surface trap states, leading to the reduction of PL QY of the crosslinked films ${ }^{48}$. Given that the reaction of azide radicals with the surface of QDs is primarily responsible for the drawback, optimising the content of LiXer in QD films is acute in achieving well-defined QD patterns with high luminance efficiency. Figure 4a shows PL QYs of 13-nm sized red-emitting CdSe/CdZnS QD films as a function of added LiXer contents. QD films prepared with LiXer contents below 2 wt $\%$ are readily devastated during the rinsing processes (Fig. $4 \mathrm{~b}$ ). LiXer contents $>2 \mathrm{wt} \%$ are sufficient to form a robust network of QDs, but high LiXer contents accompany PL QY loss of QD films during the photocrosslinking (Fig. 4a). To avoid unwanted outcome by the presence of excess azide radicals, we cut the content of LiXer in QD solutions down to the limit that ensures the structural robustness of QD films against rinsing steps. The optimum LiXer content varies depending on the QD dimension; greater LiXer contents for smaller QDs and smaller LiXer contents for larger QDs
(Fig. 4c and corresponding PL decay curves in Supplementary Fig. 8). QD films with the optimum content of LiXer retain their PL spectra (peak emission wavelength and the spectral linewidth) and PL QY throughout the photo-crosslinking and the rinsing steps (Fig. $4 \mathrm{~d}, \mathrm{e}$ ), representing that the present approach is indeed non-destructive; the photophysical properties of the QD materials could be well-preserved. Resulting QD films emit coloursaturated primary colours solely originating from the band-edge transition in QDs. We note that the colour space achieved with the resulting $R G B$ spectra of crosslinked QD films far surpasses the standards of the up-to-date commercial displays (sRGB or DCI-P3) (Fig. 4f).

Electroluminescence characteristics of crosslinked QDs with LiXer. As an ultimate achievement, we exemplify QD-LEDs with the photo-crosslinked QD films. The devices were constructed in an inverted structure employing hybrid charge transport layers (Fig. 5a) ${ }^{16}$. For effective electron injection from the indium tin oxide (ITO) cathode to the QD layer, a transparent zinc oxide nanoparticle ( $\mathrm{ZnO} \mathrm{NP}$ ) layer was adopted as an electron transport layer (ETL). The 4,4-bis(N-carbazolyl)-1,1-biphenyl (CBP) layer was used as the hole transport layer (HTL) for holes injected from the $\mathrm{MoO}_{3} / \mathrm{Al}$ anode. A $30 \mathrm{~nm}$-thick crosslinked QD emissive layer was prepared from red-emitting $\mathrm{CdSe} / \mathrm{CdZnSe} / \mathrm{ZnSeS}$ QD (diameter $=20 \mathrm{~nm}$ ) dispersion containing $1 \mathrm{wt} \%$ of LiXer. Figure $5 b$ shows a cross-sectional TEM image of the QD-LED and Fig. $5 c$ depicts the energy diagram of the device at a static condition $\left(V_{\text {applied }}=0 \mathrm{~V}\right)$. We note that the deposition of the QD emission layer, UV crosslinking and rinsing, were all carried out in an inert atmosphere. Figure $5 \mathrm{~d}-\mathrm{f}$ compare the performances of QD-LEDs incorporating a pristine QD layer (black) versus a 

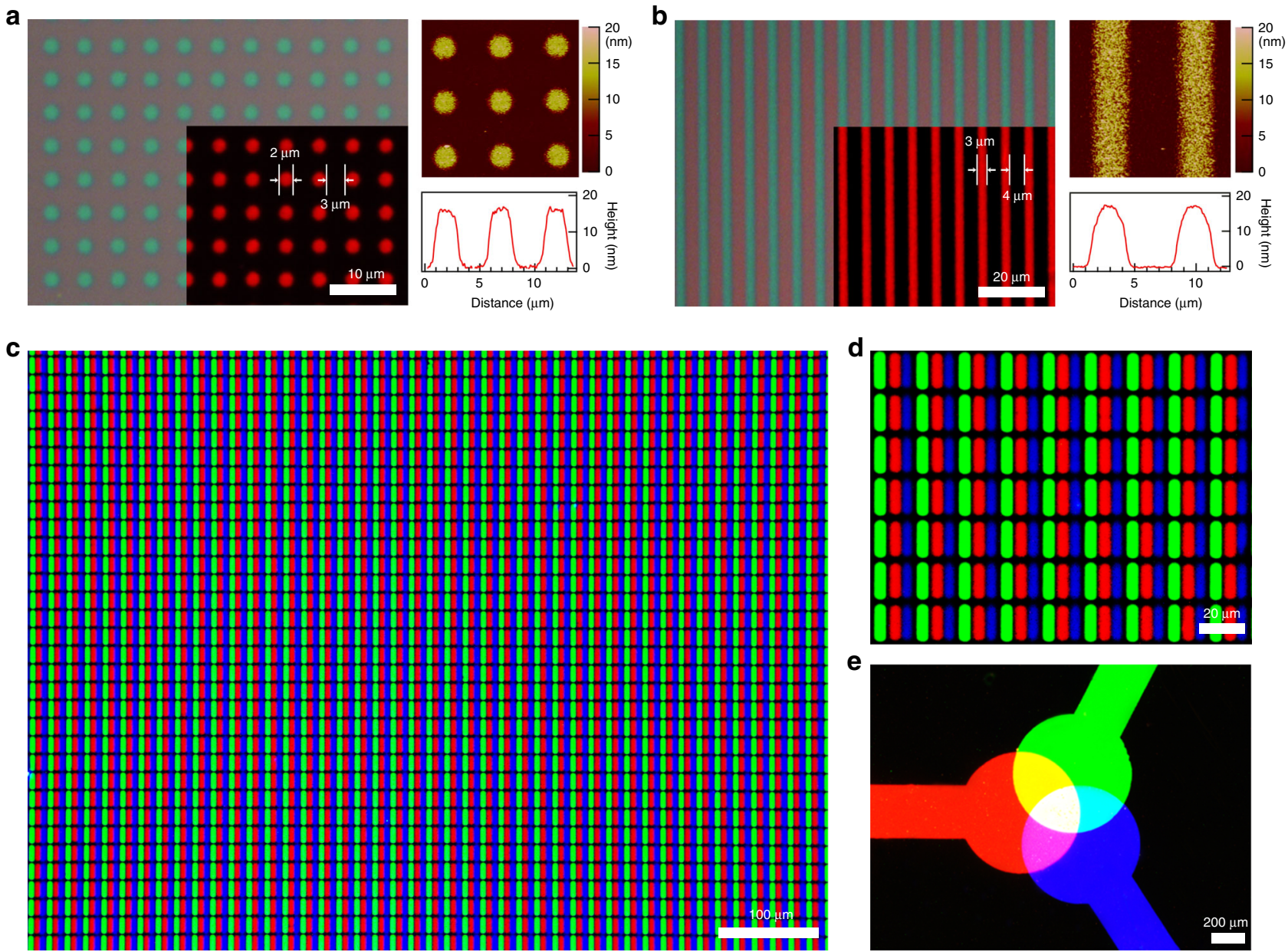

Fig. 3 Optical and fluorescence images of QD patterns. Optical and fluorescence (inset) microscopic images and AFM images with height profile of a dot (diameter $=2 \mu \mathrm{m}$, spacing $3 \mu \mathrm{m}$ ) and $\mathbf{b}$ line patterns (width $=3 \mu \mathrm{m}$, spacing $4 \mu \mathrm{m}$ ) based on 13-nm sized red-emitting CdSe/CdZnS QDs. c-e Fluorescence

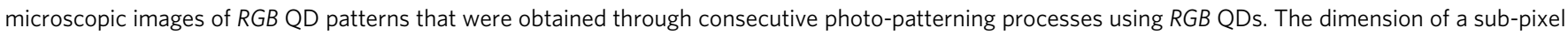
in Fig. 3c, d is $4 \mu \mathrm{m} \times 16 \mu \mathrm{m}$, corresponding to a resolution $>1400$ p.p.i. Fig. $3 \mathrm{~d}$ is a magnified view of Fig. $3 c$.

crosslinked QD layer (red). Apparently, no significant difference was observed in the electrical and optoelectronic characteristics of devices. Specifically, the turn-on voltage $\left(V_{\mathrm{T}}\right.$, defined as the voltage yielding at 1 nit) and the peak E.Q.E. of both red-emitting devices were identical as $2.4 \mathrm{~V}$ and $14.6 \%$, respectively. (Fig. $5 \mathrm{~d}$, e). The peak position of EL spectra $\left(\lambda_{\mathrm{EL}, \max }\right)$ and full-width-at-half-maximum (FWHM) of the EL spectra for both the pristine and crosslinked QD-LEDs also matched well. The performance of green- and blue-emitting QD-LEDs, as well as their EL spectra could also be preserved upon exploiting the crosslinked emissive layer (Supplementary Fig. 9). In addition, both red-emitting devices displayed similar device operation stability under the same applied current density $\left(30 \mathrm{~mA} \mathrm{~cm}^{-2}\right)$ (Fig. 5f). These results coherently attest that the emission and electrical characteristics of the QD films are well-preserved even after they are photo-crosslinked, and hence imply that the present approach is indeed applicable to nearly all optical and optoelectronic applications requiring patterned QD arrays. Finally, $R G$ pixelated QD-LED were successfully fabricated through consecutive the photo-crosslinking process. (inset in Fig. 5f) The dimension of a single rectangular pattern in the image is $10 \mu \mathrm{m} \times 38 \mu \mathrm{m}$. This result demonstrates the possibility of realising QD EL displays using LiXer.

\section{Discussion}

A straightforward and effective photo-patterning method of colloidal QDs is reported. QD films with LiXer, which is designed to crosslink the alkyl ligands of neighbouring QDs in films upon exposure to UV, are awarded to retain their structure against exposure to subsequent solution processes. Well-defined QD patterns with feature sizes of a few micrometres are, thus, easily attained by conducting the process cycle of QD/LiXer film deposition, UV exposure and rinsing. Ultimately, tandem processing of the present method permits us to achieve laterally resolved $R G B$ QD patterns (>1400 p.p.i.), as well as vertically stacked $R G B$ layers at a high resolution. Unlike previous photopatterning methods, suppression in the PL characteristics of QDs during the patterning process and the associated EL characteristics of the QD-LEDs can be avoided. As only mixing between QDs and LiXer is required, the method should be valid to nearly all QDs that entail alkyl ligands without any delicate secondary steps to prepare a precursor solution for patterning. Thus, the highest quality QDs, holding high luminescence efficiency prepared from state-of-the-art synthetic methods, should be easily applicable to this method. Overall, the approach here is expected to catalyse the practicable use of QDs in production of highresolution, large-area down-conversion or EL displays.

\section{Methods}

Materials for synthesis. Zinc acetate $\left(\mathrm{Zn}(\mathrm{ac})_{2}, 99.99 \%\right)$, sulphur (S, 99.99\%), selenium (Se, 99.99\%), oleic acid (OA, 99\%) and 1-octadecene (ODE, 99\%) were purchased from Uniam. $n$-Trioctylphosphine (TOP, technical grade, 90\%), 1 dodecanethiol (DDT, 98\%), ethylene glycol and dichloromethane (anhydrous, 

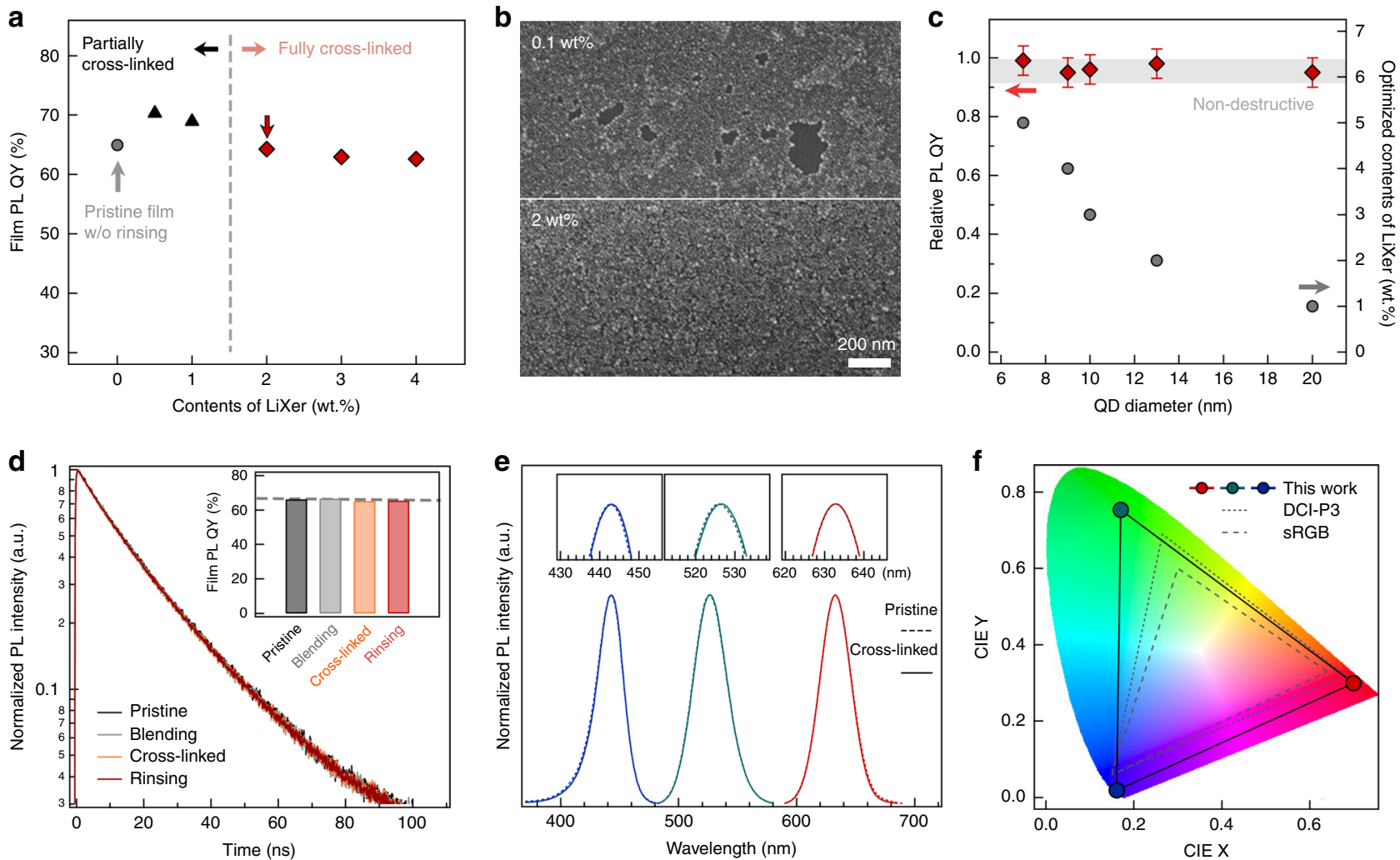

Fig. 4 Photophysical properties of crosslinked QD films. a Film PL QYs of 13-nm sized red-emitting CdSe/CdZnS QD films as a function of added LiXer contents. b SEM images of crosslinked 13-nm sized red CdSe/CdZnS QD films with 0.1 wt\% (top) and 2 wt\% (bottom) LiXer after rinsing. c The optimum LiXer contents for different sized red-emitting QDs (grey circle) and relative PL QYs of crosslinked QD films prepared with the optimum LiXer contents (red square) in comparison with pristine QD films. Error bar represents the standard deviation of the data collected from $>5$ samples prepared independently. d PL decay curves of 13-nm sized red-emitting CdSe/CdZnS QD films with 2 wt\% of LiXer throughout the film spin-casting (grey), crosslinking (orange) and rinsing (red) steps. PL decay curve of the pristine QD film (black) is overlaid for comparison. Inset: film PL QYs of each films. Each decay curve was normalised to its maximum count value. e PL spectra of pristine (dashed line) and crosslinked (solid line) red-, green- and blue-QD films. The PL QY for the pristine red-, green- and blue-QD films is $65 \%, 64 \%$ and $80 \%$, respectively. Each spectrum was normalised to its maximum PL intensity. Insets expand each spectrum at their peaks. f The colour coordinates of red-, green- and blue- QD films in the $1931 \mathrm{CIE}$ chromaticity diagram along with standard colour spaces (sRGB, DCI-P3).

$>99.8 \%$ ) were purchased from Sigma Aldrich. Myristic acid (MA, 90\%), cadmium oxide (CdO, 99.9\%) were purchased from Alfa Aesar. 4-Azido-2,3,5,6-tetrafluorobenzoic acid (>98\%) and triethyl amine (TEA, 99\%) were purchased from TCI. All chemicals are used without further purification. Thionyl chloride and the rest of other solvents were purchased from Daejung and used as received.

Materials for device fabrication. 4,4-Bis(N-carbazolyl)-1,1-biphenyl (CBP, $99.9 \%)$ were purchased from OSM. Molybdenum oxide $\left(\mathrm{MoO}_{3}, 99.95 \%\right)$ and aluminium (Al, 99.999\%) metal source were purchased from Taewon Scientific Co. (TASCO).

Synthesis of LiXer. The synthesis of LiXer (ethane-1,2-diyl bis(4-azido-2,3,5,6tetrafluorobenzoate)) was done by modifying the process described previously by Keana and colleagues ${ }^{41}$. 4-Azido-2,3,5,6-tetrafluorobenzoic acid $(931.6 \mathrm{mg}, 3.9626$ $\mathrm{mmol})$ and thionyl chloride (1 M in dichloromethane) $(23.8 \mathrm{~mL}, 23.77 \mathrm{mmol})$ were dissolved in anhydrous dichloromethane $(30.6 \mathrm{~mL})$, and this solution was heated for $19 \mathrm{~h}$ at $70^{\circ} \mathrm{C}$. The reaction mixture was cooled down to room temperature, and then the organic solvents were removed by distillation at a reduced pressure. The resulting acyl chloride compound was dissolved in anhydrous dichloromethane $(12 \mathrm{~mL})$ and transferred dropwise to a mixture of ethylene glycol $(102.48 \mathrm{mg}$, $1.6511 \mathrm{mmol})$ and TEA $(400.975 \mathrm{mg}, 0.8507 \mathrm{mmol})$ in anhydrous dichloromethane $(18 \mathrm{~mL})$. This reaction mixture was stirred for $12 \mathrm{~h}$ at room temperature and quenched by the addition of $1-\mathrm{M} \mathrm{HCl}_{(\mathrm{aq})}(25 \mathrm{~mL})$. The resulting solution was extracted with dichloromethane $(16 \mathrm{~mL} \times 3)$. The dichloromethane phases were washed with brine $(60 \mathrm{~mL})$ and dried over anhydrous $\mathrm{MgSO}_{4}$. After filtration, the organic solvent was removed from the filtrate using a rotary evaporator at a reduced pressure. The obtained crude product was purified by silica gel column chromatography, using an ethyl acetate/ $n$-hexane $(1 / 5$ to $1 / 3)$ eluent. Pure white solid of LiXer was obtained (439 mg, $54 \%) .{ }^{1} \mathrm{H}-\mathrm{NMR}\left(400 \mathrm{MHz}, \mathrm{CDCl}_{3}\right): \delta=4.68$ $(\mathrm{s}, 4 \mathrm{H}) ;{ }^{19} \mathrm{~F}-\mathrm{NMR}\left(376 \mathrm{MHz}, \mathrm{CDCl}_{3}\right): \delta=-138.17$ to $-138.26(\mathrm{~m}),-150.71$ to $-150.81(\mathrm{~m}) ;{ }^{13} \mathrm{C}-\mathrm{NMR}\left(100 \mathrm{MHz}, \mathrm{CDCl}_{3}\right): \delta=159.11-159.01,146.90-146.67$, 144.33-144.10, 141.82-141.58, 139.32-139.09, 123.96-123-72, 107.09-106.79, 63.39; gas chromatography (GC)/mass spectroscopy (MS) calculated for

$\mathrm{C}_{16} \mathrm{H}_{4} \mathrm{~F}_{8} \mathrm{~N}_{6} \mathrm{O}_{4} \mathrm{M}^{+}: 496.0166$, found: $m / z$ 496.1. Nuclear magnetic resonance (NMR) and GC/MS spectra of LiXer are given in Supplementary Information. (Supplementary Figs. 10-14)

Synthesis of red-emitting QDs. Stock solutions of $0.5-\mathrm{M}$ cadmium oleate (Cd $(\mathrm{OA})_{2}$ ) diluted in $\mathrm{ODE}$, 0.5-M zinc oleate $\left(\mathrm{Zn}(\mathrm{OA})_{2}\right)$ in ODE, 2-M TOPSe and 2-M TOPS were prepared for use in the synthesis of QDs. CdSe/CdZnS QDs were synthesised referring to the method used by Lim et al ${ }^{49}$ with minor modification For a typical synthesis of CdSe/CdZnS QDs, a mixture of $1 \mathrm{mmol}$ of $\mathrm{CdO}, 3 \mathrm{mmol}$ of MA and $15 \mathrm{~mL}$ of ODE were degassed at $110^{\circ} \mathrm{C}$ for $2 \mathrm{~h}$, followed by heating to $270^{\circ} \mathrm{C}$ to form a clear solution. Meanwhile, $0.25 \mathrm{~mL}$ of 2-M TOPSe solution was rapidly injected into the reaction flask and reacted for $3 \mathrm{~min}$ at $300^{\circ} \mathrm{C}$ to the formation of CdSe cores. To grow the CdZnS shell, $4 \mathrm{~mL}$ of $0.5-\mathrm{M} \mathrm{Zn}(\mathrm{OA})_{2}$ solution was added to the flask before $1.5 \mathrm{mmol}$ of DDT was added dropwise. After 30 min of reaction at the elevated temperature, $\mathrm{Cd}(\mathrm{OA})_{2}, \mathrm{Zn}(\mathrm{OA})_{2}$ and TOPS (total $8 \mathrm{mmol}, 17 \mathrm{mmol}$ and $23 \mathrm{mmol}$, respectively) were injected repeatedly to grow CdZnS shell in a desired thickness. Synthesised QDs was purified repeatedly via typical precipitation/redispersion method over ten times with various anti-solvents (e.g., ethanol and acetone) before use. Weakly bound TOP on the surface QDs is likely to be removed during this step. 20-nm sized CdSe/CdZnSe/ZnSeS QDs with composition gradient in the two shell layers were synthesised referring to the method used by Lim et al. ${ }^{18} \mathrm{CdZnSe}$ shell was grown on CdSe cores by continuous injection of $\mathrm{Cd}(\mathrm{OA})_{2}, \mathrm{Zn}(\mathrm{OA})_{2}$ and TOPSe. CdSe cores were prepared via the method analogous to the recipe for the core of CdSe/CdZnS QDs. For growth of CdZnSe shell, $56 \mathrm{~mL}$ of $0.5-\mathrm{M} \mathrm{Zn}(\mathrm{OA})_{2}$ solution was added to the flask followed by the continuous injection of a mixture of $12 \mathrm{~mL}$ of $0.5-\mathrm{M} \mathrm{Cd}(\mathrm{OA})_{2}$ solution, $12 \mathrm{~mL}$ of 2-M TOPSe solution and $24 \mathrm{~mL}$ of ODE with injection rate of $24 \mathrm{~mL} \mathrm{~h}^{-1}$. Additional ZnSeS shell was grown on the exterior of CdSe/CdZnSe QDs by 
a

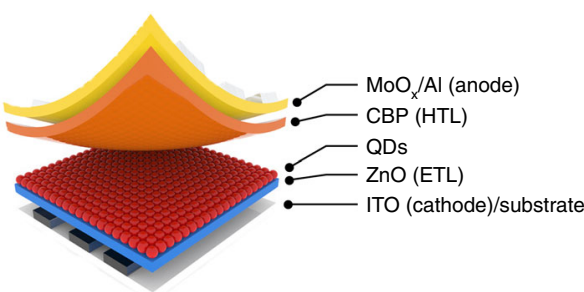

d

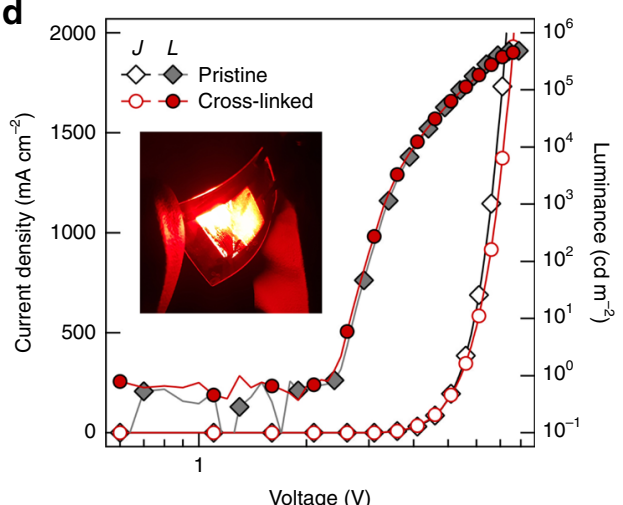

b

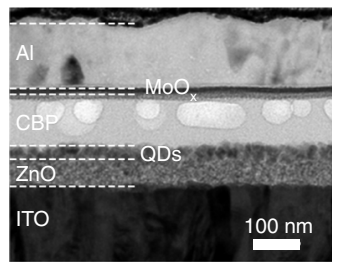

C

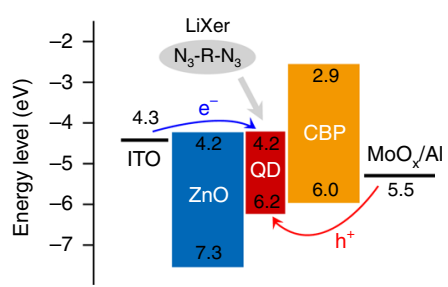

e

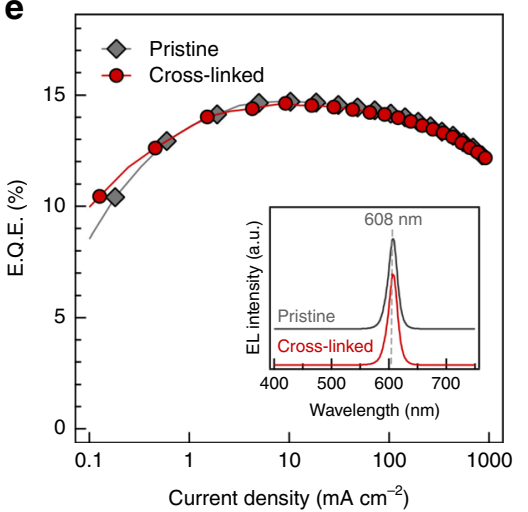

f

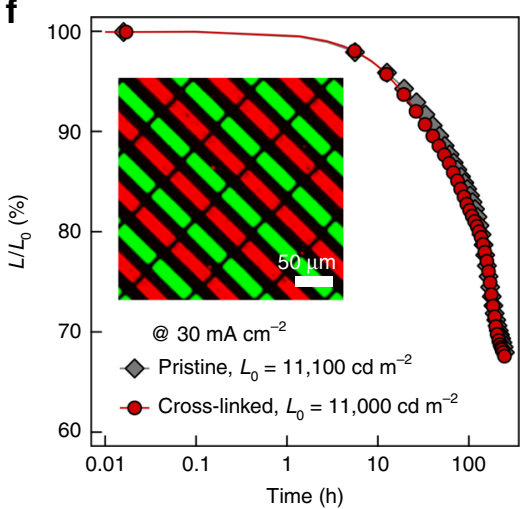

Fig. 5 Device characteristics of QD-LEDs with the crosslinked QD emissive layer. a Schematic illustration, $\mathbf{b}$ cross-sectional TEM image and $\mathbf{c}$ the energy band diagram of a representative QD-LED employing the crosslinked QD emissive layer. d Current density (J)-voltage (V)-luminance (L), e external quantum efficiency (E.Q.E.)-J and $\mathbf{f}$ temporal change in relative luminance of QD-LEDs employing pristine (grey diamond) and crosslinked (red circle). $L_{0}$ is the luminance at initial. The inset in Fig. $5 \mathrm{~d}$ is a photograph of a working device prepared on a flexible substrate. The inset in Fig. $5 \mathrm{e}$ shows EL spectra of each device. The EL spectrum of a pristine QD-LED is vertically shifted for visual clarity. The inset in Fig. $5 f$ is an EL image of a pixelated RG QD-LED (the electrodes as well as the charge injection layers were used in common to operate the device with photo-patterned QD layer). The size of a single rectangular pattern in the image is $10 \mu \mathrm{m} \times 38 \mu \mathrm{m}$.

continuous injection of $80 \mathrm{mmol}$ of $\mathrm{Zn}(\mathrm{OA})_{2}, 32 \mathrm{mmol}$ of TOPSe and $34 \mathrm{mmol}$ of TOPS. Synthesised CdSe/CdZnSe/ZnSeS QDs were purified repeatedly via typical precipitation/redispersion method. Synthetic schemes for green and blue coloured QDs are detailed in Supplementary Methods.

Device fabrication. Photo-patterned QD-LEDs were fabricated in an inverted structure. Typical processes are described as follows. Glass substrates pre-patterned with indium tin oxide (ITO) electrodes were first cleaned with isopropyl alcohol, acetone and distilled water in an ultrasonic bath for $15 \mathrm{~min}$ each. Then, $20 \mathrm{mg} \mathrm{mL}^{-1}$ of $\mathrm{ZnO}$ NPs dispersed in 1-butanol was spin-coated onto the substrate at $2000 \mathrm{rpm}$ for $40 \mathrm{sec}$, and the films were annealed at $100^{\circ} \mathrm{C}$ for $30 \mathrm{~min}$ under a nitrogen atmosphere. Dispersions of QDs in toluene added with different contents of LiXer were spin-coated at $4000 \mathrm{rpm}$ for $30 \mathrm{sec}$. The resulting films were irradiated with a handheld UV-light source $\left(254 \mathrm{~nm}, 0.4 \mathrm{~mW} \mathrm{~cm}^{-2}\right)$ for $5 \mathrm{sec}$ to derive the crosslinking reaction between QDs and LiXer. After the irradiation process, the films were developed by rinsing the films with toluene. This process effectively removed the QDs in the areas not exposed to UV (Supplementary Fig. 15). CBP (60 nm), $\mathrm{MoO}_{3}(10 \mathrm{~nm})$ and $\mathrm{Al}(130 \mathrm{~nm})$ were consecutively deposited using a thermal evaporator.

Characterisation. ${ }^{1} \mathrm{H}-\mathrm{NMR},{ }^{13} \mathrm{C}-\mathrm{NMR}$ and ${ }^{19} \mathrm{~F}-\mathrm{NMR}$ were measured by a Bruker Avance III HD at 400, 100 and $376 \mathrm{MHz}$, respectively, with deuterated chloroform $\left(\mathrm{CDCl}_{3}\right)$ as solvent, which was purchased from Cambridge Isotope Laboratories. GC/MS was measured by a Bruker $450-\mathrm{GC}$ and $320-\mathrm{MS}$ at the UNIST Central Research Facilities Centre (UCRF), Ulsan, Korea. UV-Vis absorption and fluorescence spectra of QD films were measured using a V-770 UV-Vis-NIR spectrophotometer (Jasco) and FS-2 fluorescence spectrometer (Scinco), respectively. The measurements of time-resolved photoluminescence (TRPL) were conducted with time-correlated single photon counting (TCSPC) system from Horiba-Jovin Yvon with resolution of about $100 \mathrm{ps}$. The samples were excited at $3.06 \mathrm{eV}$ at a repetition rate of $500 \mathrm{kHz}$. FT-IR spectra were collected in a reflectance mode under ambient condition using an iS10 FT-IR spectrometer (Thermo Fisher Scientific). Samples for FT-IR analysis were prepared on bare Si wafer substrates. AFM images were taken with a Park NX10 AFM system (Park Systems) under ambient conditions through a non-contact mode. The thickness of the crosslinked QD films were measured using a Dektak XT-E stylus profiler. X-ray diffraction (XRD) measurements were performed using an Ultima IV X-ray diffractometer (Rigaku) $(\lambda \mathrm{CuK} \alpha 1=1.5406 \AA, 40 \mathrm{kV}, 30 \mathrm{~mA})$. Transmission electron microscopy (TEM) images were collected using a JEM-3010 (JEOL) equipped with a Gatan digital camera (MSC-794) (point resolution of $0.17 \mathrm{~nm}$ ) at the National Centre for Inter-University Research Facilities (NCIRF), Seoul National University. Scanning electron microscopy (SEM) images were obtained using a GeminiSEM 300 (Zeiss). Electrical characteristics of the photo-crosslinked QD films were analysed using a Keithley 236 source measure unit and a Keithley 2000 multimeter connected to calibrated Si photodiodes. Electroluminescence (EL) spectra were measured with a Konica-Minolta CS-2000 spectroradiometer. High-resolution single and multicolour patterns of QDs were fabricated by using a Karl Süss MA-6 Mask Aligner. Grazing incidence small-angle X-ray scattering (GI-SAXS) measurements were performed at the 9 A U-SAXS beamline of Pohang Light Source-II (PLS-II) in Republic of Korea. The X-ray coming from the in-vacuum undulator (IVU) are monochromated using Si (111) double crystals and focused at the detector position using K-B type mirror. Two-dimensional (2D) scattering patterns were recorded with a 2D CCD (Rayonix MX170-HS). The wavelength of X-ray and sample-todetector distance were set to be $1.12 \AA$ and $2.5 \mathrm{~m}$, respectively.

\section{Data availability}

All data generated or analysed during this study are included in this article (and its Supplementary Information files).

Received: 15 December 2019; Accepted: 12 May 2020;

Published online: 08 June 2020

\section{References}

1. Ekimov, A. I., Efros, A. L. \& Onushchenko, A. A. Quantum size effect in semiconductor microcrystals. Solid State Commun. 56, 921-924 (1985).

2. Brus, L. Electronic wave functions in semiconductor clusters: experiment and theory. J. Phys. Chem. 90, 2555-2560 (1986).

3. Alivisatos, A. P. Semiconductor clusters, nanocrystals, and quantum dots. Science 271, 933-937 (1996).

4. Norris, D. J. \& Bawendi, M. G. Measurement and assignment of the sizedependent optical spectrum in CdSe quantum dots. Phys. Rev. B 53, 16338-16346 (1996). 
5. Hines, M. A. \& Guyot-Sionnest, P. Synthesis and characterization of strongly luminescing ZnS-capped CdSe nanocrystals. J. Phys. Chem. 100, 468-471 (1996).

6. Jeong, B. G. et al. Colloidal spherical quantum wells with near-unity photoluminescence quantum yield and suppressed blinking. ACS Nano 10, 9297-9305 (2016).

7. Zhang, F. et al. Super color purity green quantum dot light-emitting diodes fabricated by using CdSe/CdS nanoplatelets. Nanoscale 8, 12182-12188 (2016).

8. Jang, E. et al. White-light-emitting diodes with quantum dot color converters for display backlights. Adv. Mater. 22, 3076-3080 (2010).

9. Sun, J.-Y. et al. Facile two-step synthesis of all-inorganic perovskite $\mathrm{CsPbX}_{3}$ $(\mathrm{X}=\mathrm{Cl}, \mathrm{Br}$, and $\mathrm{I})$ zeolite-Y composite phosphors for potential backlight display application. Adv. Funct. Mater. 27, 1704371 (2017).

10. Lai, C.-F., Tien, Y.-C., Tong, H.-C., Zhong, C.-Z. \& Lee, Y.-C. Highperformance quantum dot light-emitting diodes using chip-scale package structures with high reliability and wide color gamut for backlight displays. RSC Adv. 8, 35966-35972 (2018).

11. Colvin, V. L., Schlamp, M. C. \& Alivisatos, A. P. Light-emitting diodes made from cadmium selenide nanocrystals and a semiconducting polymer. Nature 370, 354-357 (1994).

12. Coe, S., Woo, W.-K., Bawendi, M. G. \& Bulović, V. Electroluminescence from single monolayers of nanocrystals in molecular organic devices. Nature $\mathbf{4 2 0}$, 800-803 (2002).

13. Mueller, A. H. et al. Multicolor light-emitting diodes based on semiconductor nanocrystals encapsulated in GaN charge injection layers. Nano Lett. 5, 1039-1044 (2005).

14. Sun, Q. et al. Bright, multicoloured light-emitting diodes based on quantum dots. Nat. Photonics 1, 717-722 (2007).

15. Anikeeva, P. O., Halpert, J. E., Bawendi, M. G. \& Bulović, V. Quantum dot light-emitting devices with electroluminescence tunable over the entire visible spectrum. Nano Lett. 9, 2532-2536 (2009).

16. Kwak, J. et al. Bright and efficient full-color colloidal quantum dot lightemitting diodes using an inverted device structure. Nano Lett. 12, 2362-2366 (2012).

17. Dai, X. et al. Solution-processed, high-performance light-emitting diodes based on quantum dots. Nature 515, 96-99 (2014).

18. Lim, J., Park, Y.-S., Wu, K., Yun, H. J. \& Klimov, V. I. Droop-free colloidal quantum dot light-emitting diodes. Nano Lett. 18, 6645-6653 (2018).

19. Chang, J. H. et al. Unraveling the origin of operational instability of quantum dot based light-emitting diodes. ACS Nano 12, 10231-10239 (2018).

20. Won, Y.-H. et al. Highly efficient and stable InP/ZnSe/ZnS quantum dot lightemitting diodes. Nature 575, 634-638 (2019).

21. Khan, Q. et al. Structure optimization of perovskite quantum dot lightemitting diodes. Nanoscale 11, 5021-5029 (2019).

22. Rhee, S. et al. "Positive incentive" approach to enhance the operational stability of quantum dot-based light-emitting diodes. ACS Appl. Mater. Interfaces 11, 40252-40259 (2019).

23. Tian, P. F. et al. Precise, scalable shadow mask patterning of vacuumdeposited organic light emitting devices. J. Vac. Sci. Technol. 17, 2975-2981 (1999).

24. Lim, J. et al. Perspective on synthesis, device structures, and printing processes for quantum dot displays. Opt. Mater. Express 2, 594-628 (2012).

25. Kwon, J. H., Yoo, S., Lampande, R. \& Kim, S. Vacuum Deposition. in Handbook of Organic Light-Emitting Diodes (eds Adachi, C., Hattori, R., Kaji, H. \& Tsujimura, T.) (Springer Japan, 2019).

26. Shulga, A. G. et al. Patterned quantum dot photosensitive FETs for medium frequency optoelectronics. Adv. Mater. Technol. 4, 1900054 (2019).

27. Park, J.-S. et al. Alternative patterning process for realization of large-area, full-color, active quantum dot display. Nano Lett. 16, 6946-6953 (2016).

28. Wood, V. et al. Inkjet-printed quantum dot-polymer composites for full-color AC-driven displays. Adv. Mater. 21, 2151-2155 (2009).

29. Kim, B. H. et al. High-resolution patterns of quantum dots formed by electrohydrodynamic jet printing for light-emitting diodes. Nano Lett. 15, 969-973 (2015).

30. Yang, P., Zhang, L., Kang, D. J., Strahl, R. \& Kraus, T. High-resolution inkjet printing of quantum dot light-emitting microdiode arrays. Adv. Opt. Mater. 8 1901429 (2019).

31. Kim, T.-H. et al. Full-colour quantum dot displays fabricated by transfer printing. Nat. Photonics 5, 176 (2011).

32. Choi, M. K. et al. Wearable red-green-blue quantum dot light-emitting diode array using high-resolution intaglio transfer printing. Nat. Commun. 6, 7149 (2015).

33. Keum, H. et al. Photoresist contact patterning of quantum dot films. ACS Nano 12, 10024-10031 (2018).

34. Palazon, F., Akkerman, Q. A., Prato, M. \& Manna, L. X-ray lithography on perovskite nanocrystals films: from patterning with anion-exchange reactions to enhanced stability in air and water. ACS Nano 10, 1224-1230 (2016).
35. Wang, L. et al. Giant stability enhancement of $\mathrm{CsPbX}_{3}$ nanocrystal films by plasma-induced ligand polymerization. ACS Appl. Mater. Interfaces 11, 35270-35276 (2019)

36. Wang, Y., Fedin, I., Zhang, H. \& Talapin, D. V. Direct optical lithography of functional inorganic nanomaterials. Science 357, 385-388 (2017).

37. Wang, Y., Pan, J.-A., Wu, H. \& Talapin, D. V. Direct wavelength-selective optical and electron-beam lithography of functional inorganic nanomaterials. ACS Nano 13, 13917-13931 (2019).

38. Reiser, A. \& Leyshon, L. J. Spin state of photogenerated phenylnitrene. J. Am. Chem. Soc. 93, 4051-4052 (1971).

39. Leyva, E., Platz, M. S., Persy, G. \& Wirz, J. Photochemistry of phenyl azide: the role of singlet and triplet phenylnitrene as transient intermediates. J. Am. Chem. Soc. 108, 3783-3790 (1986).

40. Keana, J. F. W. \& Cai, S. X. New reagents for photoaffinity labeling: synthesis and photolysis of functionalized perfluorophenyl azides. J. Org. Chem. 55, 3640-3647 (1990)

41. Cai, S. X., Glenn, D. J., Kanskar, M., Wybourne, M. N. \& Keana, J. F. W. Development of highly efficient deep-UV and electron beam mediated crosslinkers: synthesis and photolysis of bis(perfluorophenyl) azides. Chem. Mater. 6, 1822-1829 (1994).

42. Khong, S.-H. et al. General photo-patterning of polyelectrolyte thin films via efficient ionic bis(fluorinated phenyl azide) photo-crosslinkers and their postdeposition modification. Adv. Funct. Mater. 17, 2490-2499 (2007).

43. Png, R.-Q. et al. High-performance polymer semiconducting heterostructure devices by nitrene-mediated photocrosslinking of alkyl side chains. Nat. Mater. 9, 152 (2009).

44. Wang, G.-J. N. et al. Tuning the cross-linker crystallinity of a stretchable polymer semiconductor. Chem. Mater. 31, 6465-6475 (2019).

45. Fleet, G. W. J., Porter, R. R. \& Knowles, J. R. Affinity labelling of antibodies with aryl nitrene as reactive group. Nature 224, 511-512 (1969).

46. Poe, R., Schnapp, K., Young, M. J. T., Grayzar, J. \& Platz, M. S. Chemistry and kinetics of singlet pentafluorophenylnitrene. J. Am. Chem. Soc. 114, 5054-5067 (1992).

47. Liu, L.-H. \& Yan, M. Perfluorophenyl azides: new applications in surface functionalization and nanomaterial synthesis. Acc. Chem. Res. 43, 1434-1443 (2010).

48. Giansante, C. \& Infante, I. Surface traps in colloidal quantum dots: a combined experimental and theoretical perspective. J. Phys. Chem. Lett. 8 5209-5215 (2017).

49. Lim, J. et al. Influence of shell thickness on the performance of light-emitting devices based on $\mathrm{CdSe} / \mathrm{Zn}_{1-\mathrm{x}} \mathrm{Cd} \mathrm{x}$ core/shell heterostructured quantum dots. Adv. Mater. 26, 8034-8040 (2014).

\section{Acknowledgements}

This work was supported by the Basic Science Programme (NRF-2017R1C1B2006789 and 2020R1A2C2011478), the Creative Materials Discovery Programme (NRF2019M3D1A1078299), and the Engineering Research Centre Programme (NRF2018R1A5A1025594) through the National Research Foundation of Korea (NRF) funded by the Ministry of Science and ICT, Korea.

\section{Author contributions}

M.S.K., W.K.B. and J.H.C. (Jeong Ho Cho) conceived the core strategy of the patterning method and designed the experiment. J.Y., K.K., S.K., H.W.P., M.L. (Minkyoung Lee) and H.K. carried out the patterning experiment and thin film characterisation. J.Y., K.K., S.R., J.K. and C.L. fabricated the QD-LEDs and analysed their performance. D.H., J.H.C. (Jun Hyuk Chang), and J.L. synthesised the QDs. D.H., J.H.C. (Jun Hyuk Chang) and W.K.B. analysed the luminescent characteristics of the patterned QDs. J.B. and H.A. analysed the structural characterisation of QD films. M.L. (Myeongjae Lee) and B.K. synthesised LiXer. J.Y., D.H., W.K.B. and M.S.K. mainly prepared the manuscript.

\section{Competing interests}

The authors declare no competing interests.

\section{Additional information}

Supplementary information is available for this paper at https://doi.org/10.1038/s41467 020-16652-4.

Correspondence and requests for materials should be addressed to W.K.B. or M.S.K.

Peer review information Nature Communications thanks Hunter McDaniel and the other, anonymous, reviewer(s) for their contribution to the peer review of this work.

Reprints and permission information is available at http://www.nature.com/reprints

Publisher's note Springer Nature remains neutral with regard to jurisdictional claims in published maps and institutional affiliations. 
(c) (i) Open Access This article is licensed under a Creative Commons Attribution 4.0 International License, which permits use, sharing, adaptation, distribution and reproduction in any medium or format, as long as you give appropriate credit to the original author(s) and the source, provide a link to the Creative Commons license, and indicate if changes were made. The images or other third party material in this article are included in the article's Creative Commons license, unless indicated otherwise in a credit line to the material. If material is not included in the article's Creative Commons license and your intended use is not permitted by statutory regulation or exceeds the permitted use, you will need to obtain permission directly from the copyright holder. To view a copy of this license, visit http://creativecommons.org/ licenses/by/4.0/.

(C) The Author(s) 2020 